\title{
TectoMT:
}

\section{Modular NLP Framework}

\section{Martin Popel, Zdeněk Žabokrtský ÚFAL, Charles University in Prague}

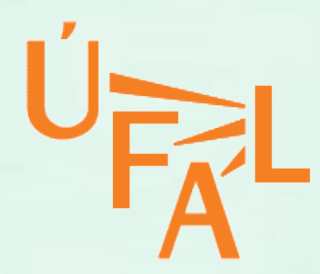

IceTAL, 7th International Conference on

Natural Language Processing August 17, 2010, Reykjavik 


\section{Outline}

- Motivation

- Layers of language description in PDT

- TectoMT architecture

- TectoMT internals

- Future plans

- Conclusion 


\section{Motivation for creating TectoMT}

Originally a framework for a linguistically motivated MT system

- deep syntactic (tectogrammatical) transfer

- started with English to Czech direction

- translation process divided to $~ 90$ "blocks"

- combining statistical and rule-based blocks

\section{Goals:}

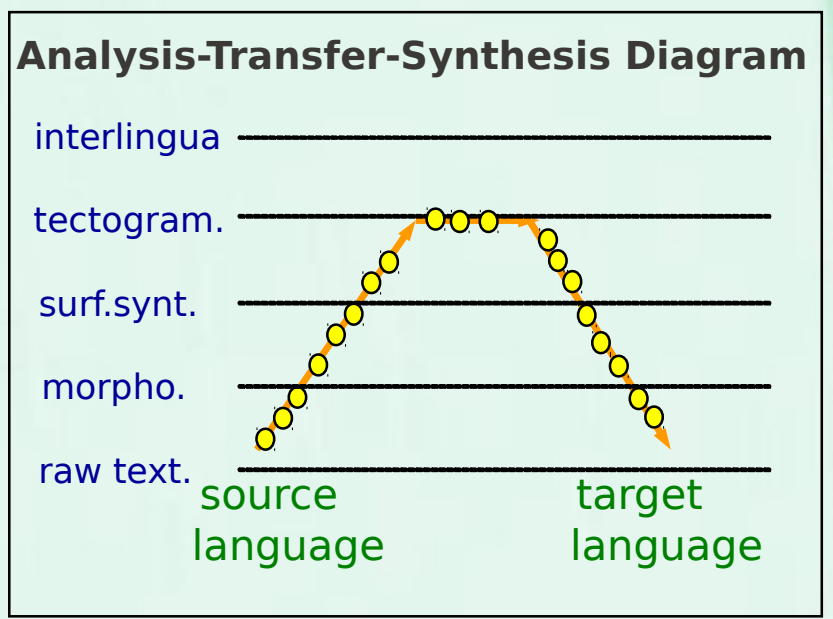

- elegant integration of in-house and third-party NLP tools

- modularity, reusability, cooperation

- ability to easily modify and add code in full-fledged programming language (Perl) 


\section{Motivation for creating TectoMT}

Now used for many other projects, not limited to MT nor tectogrammatics:

- automatic alignment \& annotation of a parallel treebank (CzEng)

- support for manual annotations (PEDT)

- lemmatization, tagging, parsing

- named entity recognition, information retrieval, coreference

- preprocessing for phrase-base MT

- change word order, append determiners to nouns,...

- add deep-syntactic features as an input for factored translation

- conversions, evaluations, etc. 


\section{4 layers of language description implemented in Prague Dependency Treebank (PDT)}

- word layer raw (tokenized) text

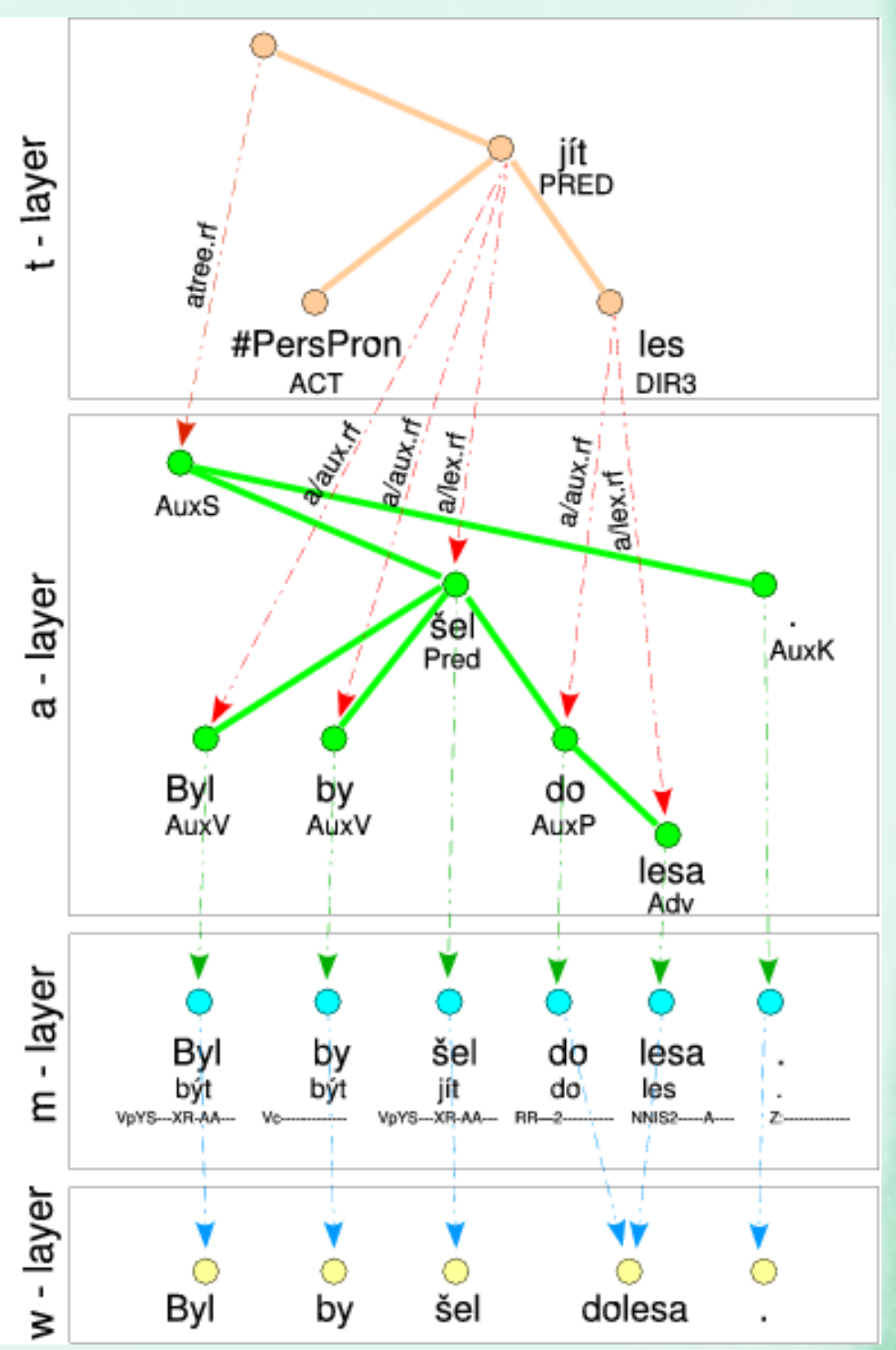




\section{4 layers of language description implemented in Prague Dependency Treebank (PDT)}

- morphological layer lemma \& POS tag for each word

- word layer raw (tokenized) text

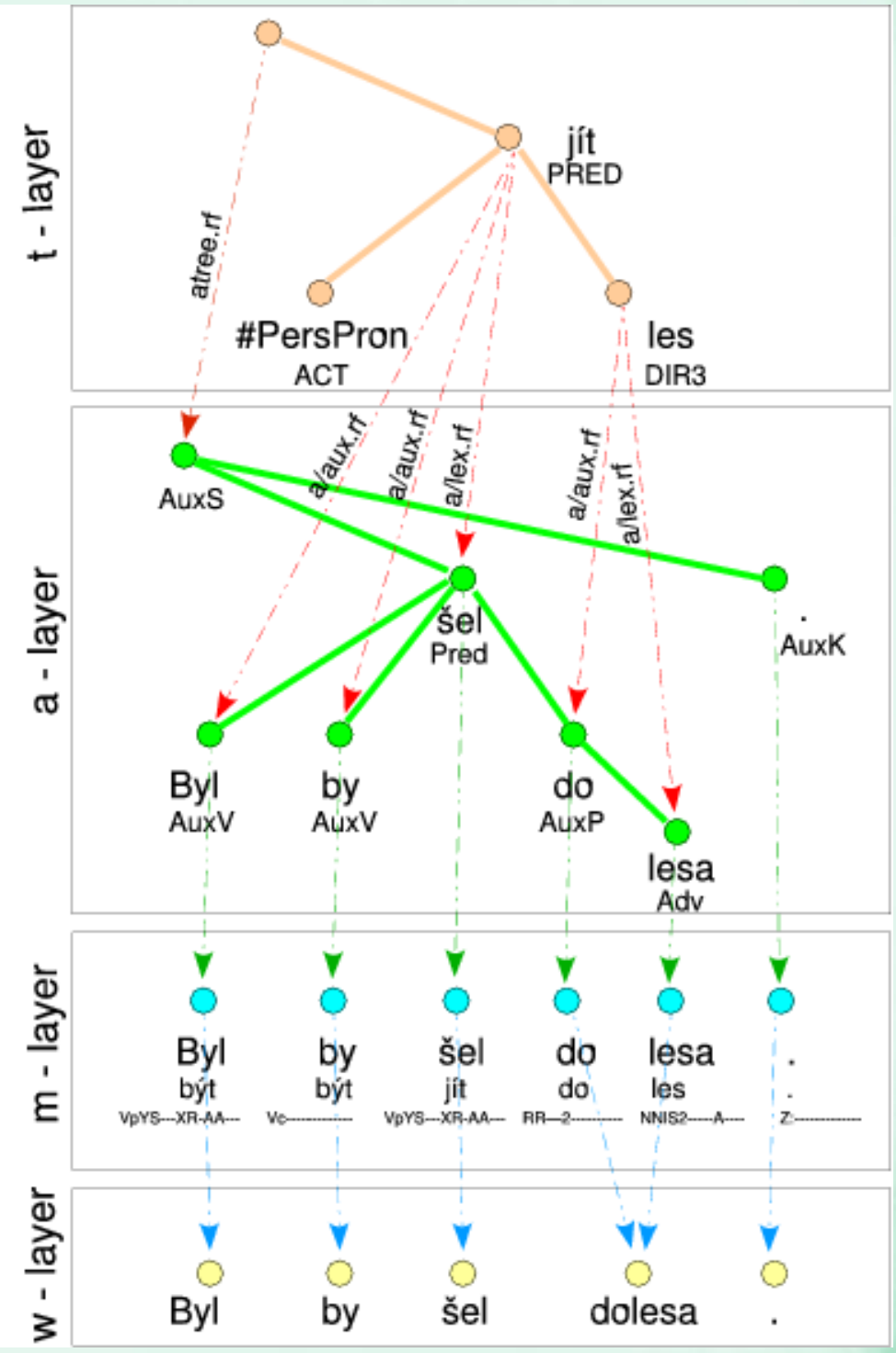




\section{4 layers of language description implemented in Prague Dependency Treebank (PDT)}

- analytical layer surface-syntactic dependency trees, labeled edges

- morphological layer lemma \& POS tag for each word

- word layer raw (tokenized) text
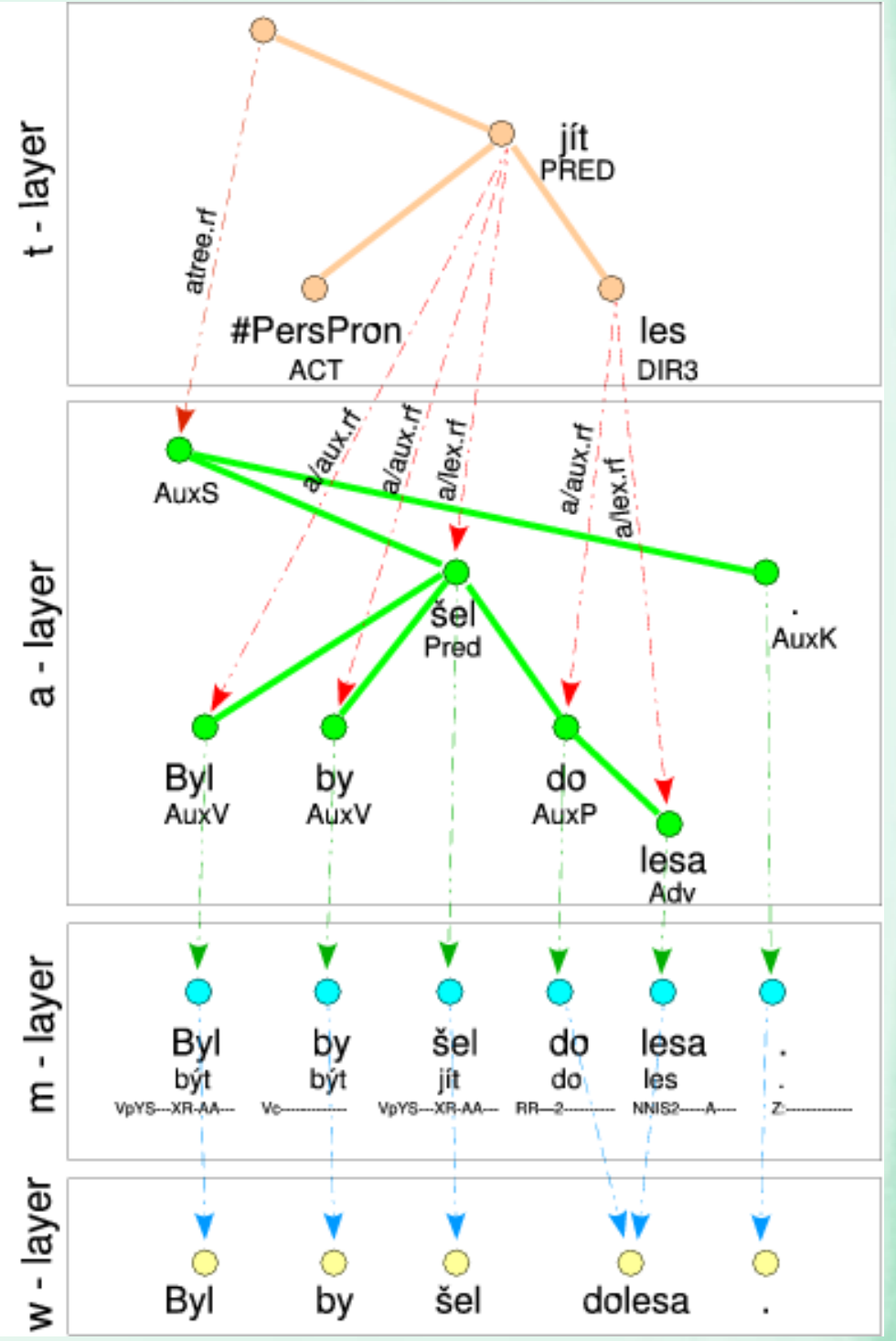


\section{4 layers of language description implemented in Prague Dependency Treebank (PDT)}

- tectogrammatical layer deep-syntactic dependency trees

- analytical layer surface-syntactic dependency trees, labeled edges

- morphological layer lemma \& POS tag for each word

- word layer

raw (tokenized) text
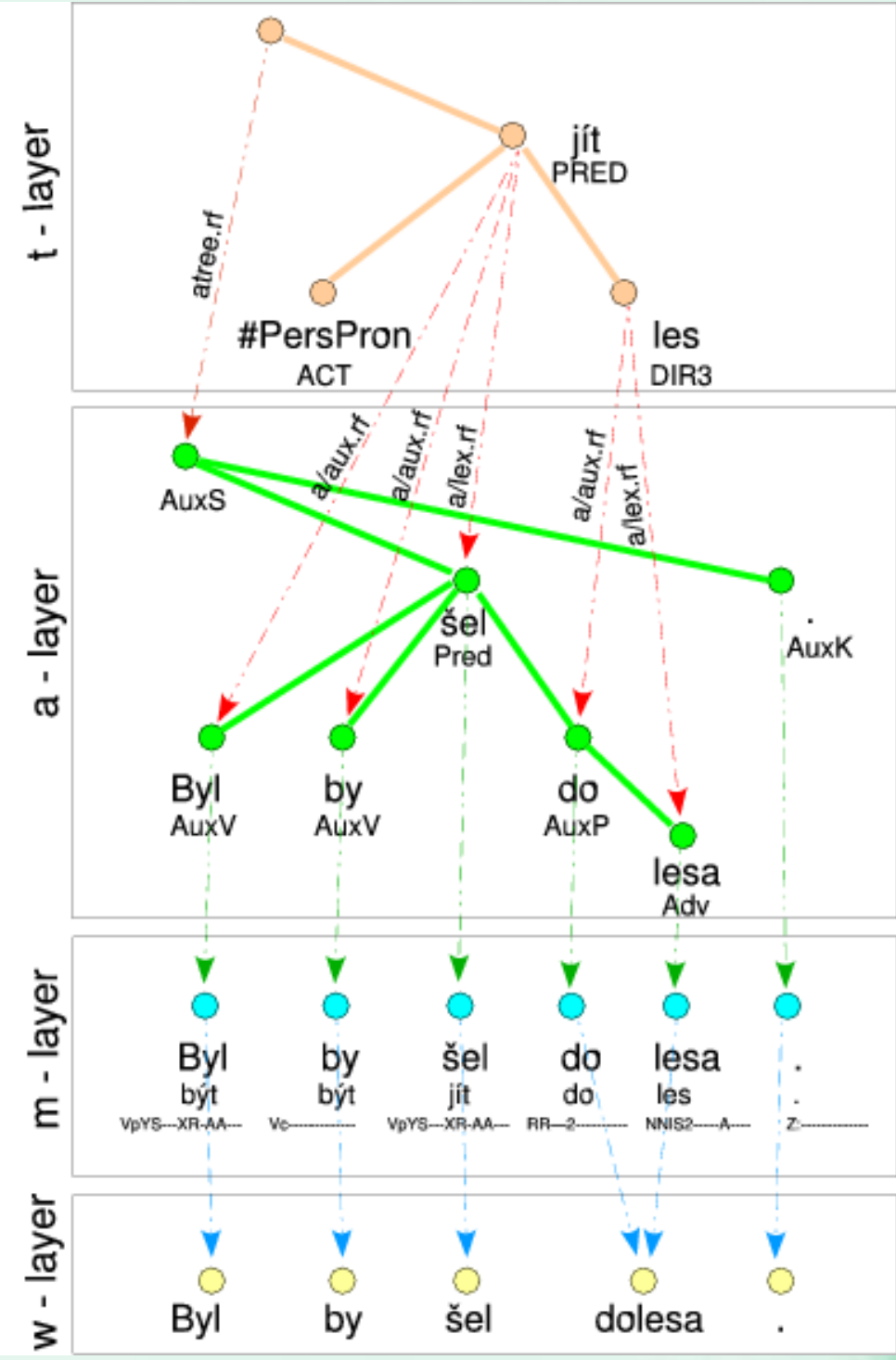


\section{4 layers of language description implemented in Prague Dependency Treebank (PDT)}

- tectogrammatical layer deep-syntactic dependency trees

- abstraction from many language-specific phenomena

- autosemantic (meaningful) words

$\sim$ nodes

- functional words (prepositions, auxiliaries)

attributes

- syntactic-semantic relations (dependecies) ح edges

- added nodes (e.g. becasue of pro-drop)
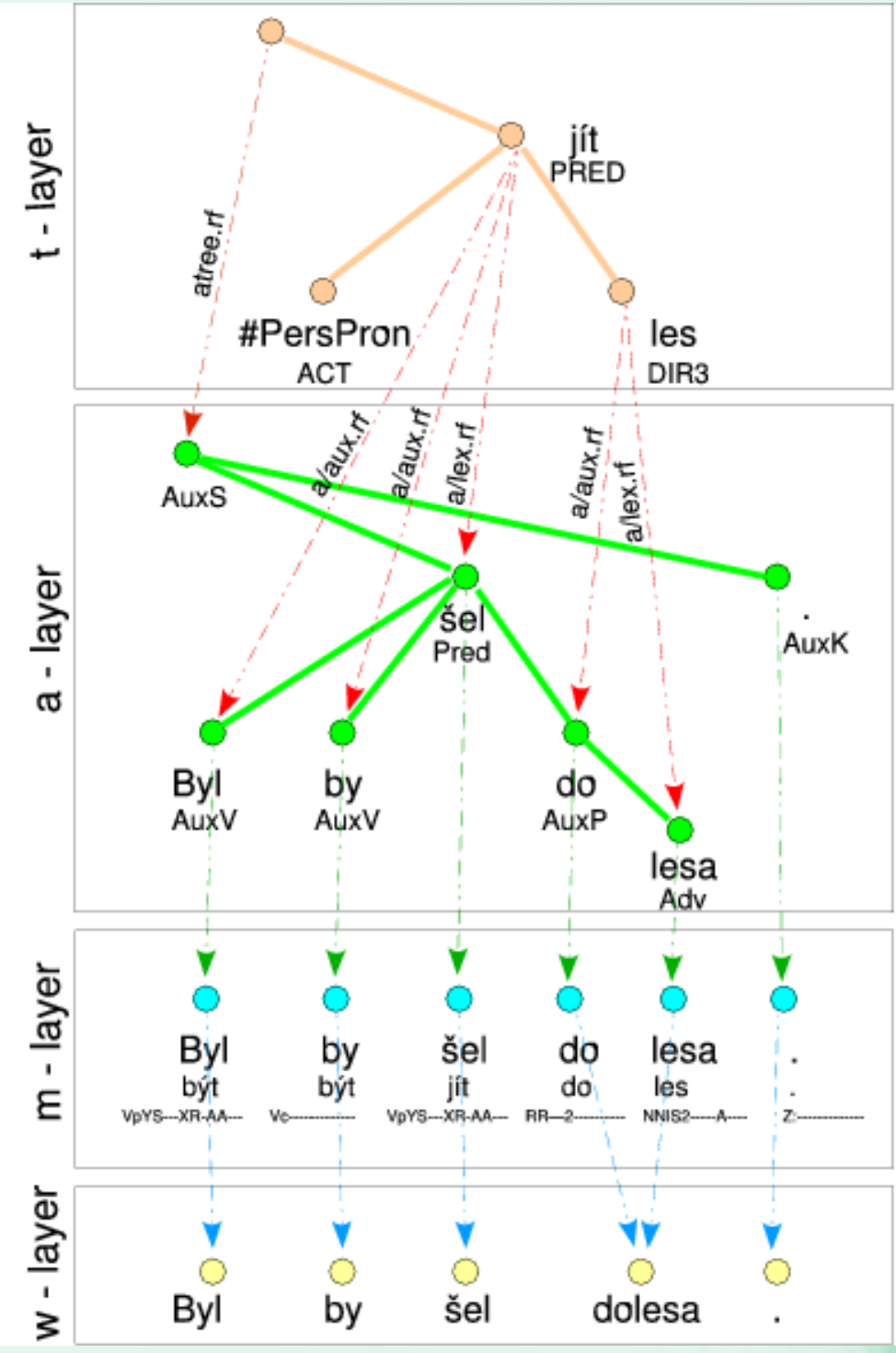


\section{TectoMT architecture processing units}

- block - elementary processing unit in TectoMT

- corresponding to a given NLP subtask

- one Perl class, saved in one file

- scenario - a sequence of blocks

- saved in plain text files

- just a list of the blocks' names and their parameters

- application - represents an end-to-end NLP task

- conversion of the input to TectoMT internal format (XML)

- possibly split into more files

- applying a scenario to the files (loaded in memory)

- conversion to the desired output format 


\section{TectoMT architecture processing units}

Blocks can be easily substituted with an alternative solution.

Scenario 1:

Scenario 2:

Scenario 3:

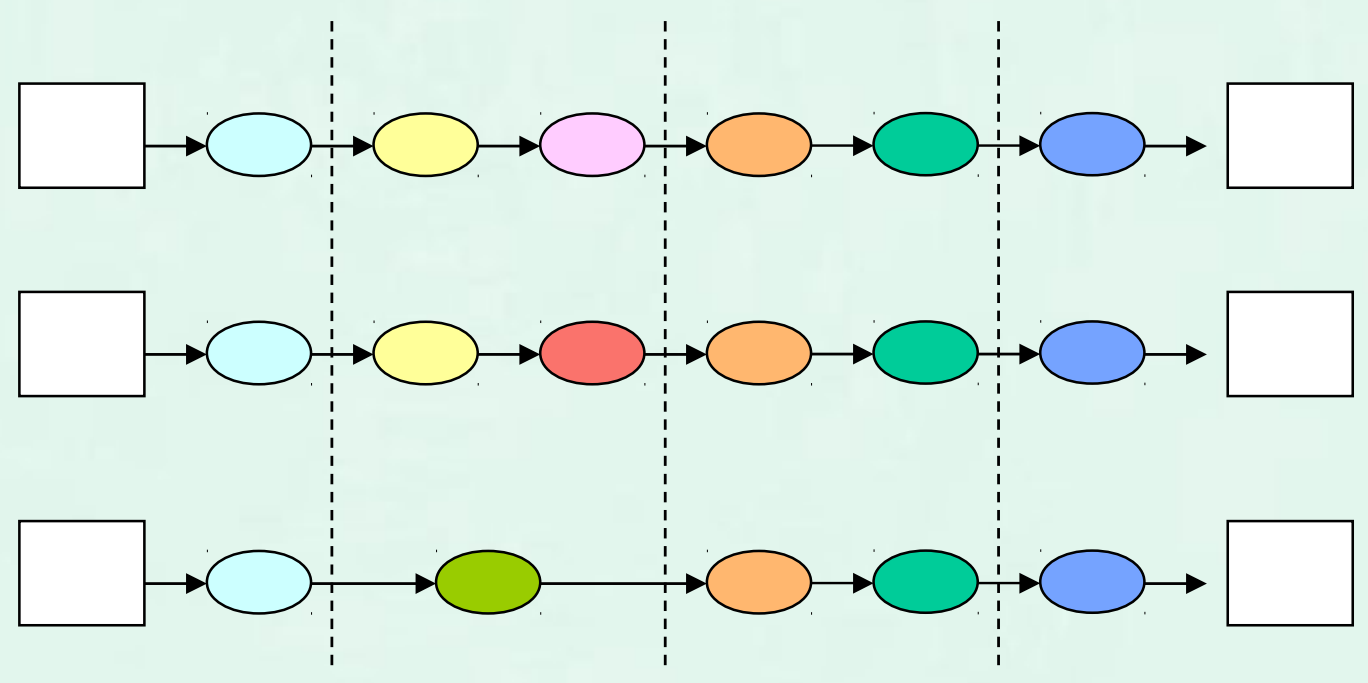




\section{TectoMT architecture processing units}

Blocks can be easily substituted with an alternative solution.

Scenario A

Sentence_segmentation_simple Penn_style_tokenization

TagMxPost

Lemmatize_mtree

McD_parser
Scenario B

Each_line_as_sentence

Tokenize_and_tag

Lemmatize_mtree

Malt_parser 


\section{TectoMT architecture data units}

- Document

- stored in one file

- sequence of sentences

- Bundle

- corresponds to one sentence

- "bundle of trees"

- Tree

- direction ( $\mathrm{S}=$ source, $\mathrm{T}=$ target)

- Language (Arabic, Czech, English, German,...)

- layer of language description (M, A, T) 


\section{TectoMT architecture data units}

\section{DOCUMENT}

sentence 1

\section{BUNDLE}

English layers

SEnglishW

Peter does not love Mary.

SEnglishM

- 00

Peter do not love Mary NNP VBZ RB VBD NNP

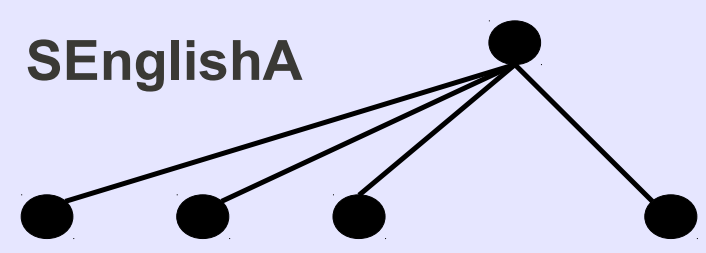

Peter do not love Mary

$\mathrm{Sb}$ AuxV Neg Pred Obj

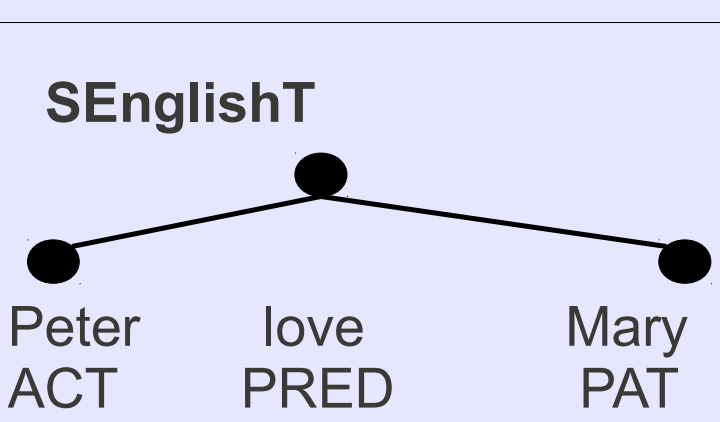

Czech layers

SCzechW

Petr nemiluje Marii.

SCzechM

Petr milovat Marie NNMS1 VB-S-3P-NA NNFS4

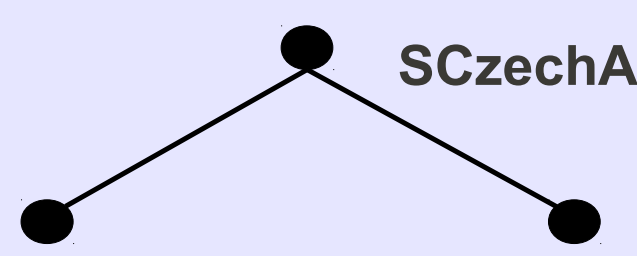

Petr milovat Marie

$\mathrm{Sb} \quad$ Pred Obj

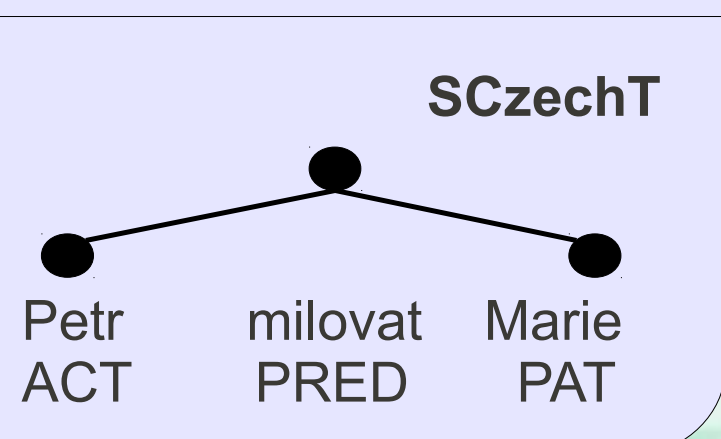

sentence 2

BUNDLE sentence $\mathrm{N}$

BUNDLE 


\section{TectoMT architecture data units}

\section{DOCUMENT}

sentence 1

English layers

\section{BUNDLE}

Czech layers

SEnglishW Peter does not love Mary.

SEnglishM

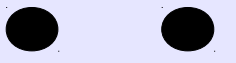

Peter do $n$ NNP VBZ $\mathrm{F}$

SEnglishA

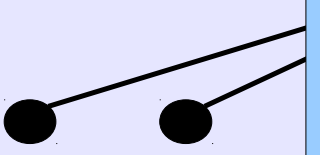

Peter do $n$

$\mathrm{Sb}$ AuxV N

SEnglishT

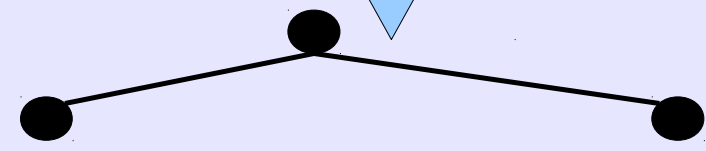

Peter ACT love PRED

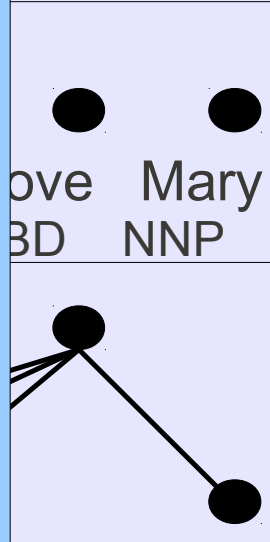

ove Mary

brad Obj
SCzechW Petr nemiluje Marii.

CzechM

Marie -NA NNFS4

SCzechA

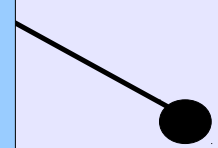

Marie

Obj

$\mathrm{Sb} \quad$ Pre

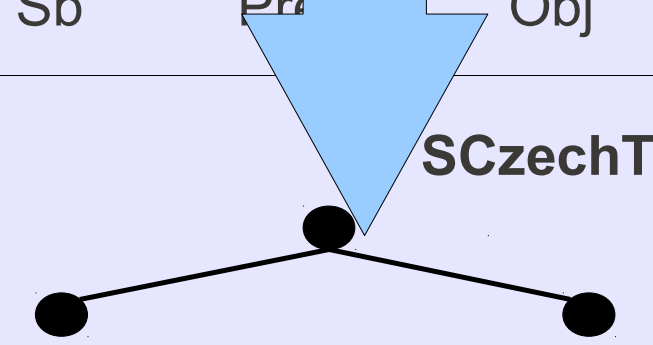

Petr milovat Marie ACT PRED PAT sentence 2

BUNDLE sentence $\mathrm{N}$

BUNDLE 


\section{TectoMT architecture data units}

\section{DOCUMENT}

sentence 1

\section{BUNDLE}

English layers

SEnglishW

Peter does not love Mary.

SEnglishM

O

Peter do not love Mary NNP VBZ RB VBD NNP

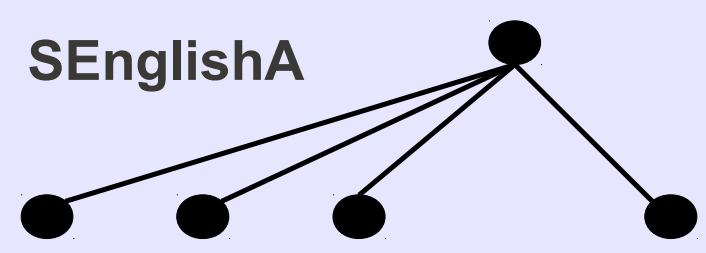

Peter do not love Mary

$\mathrm{Sb}$ AuxV Neg Pred Obj

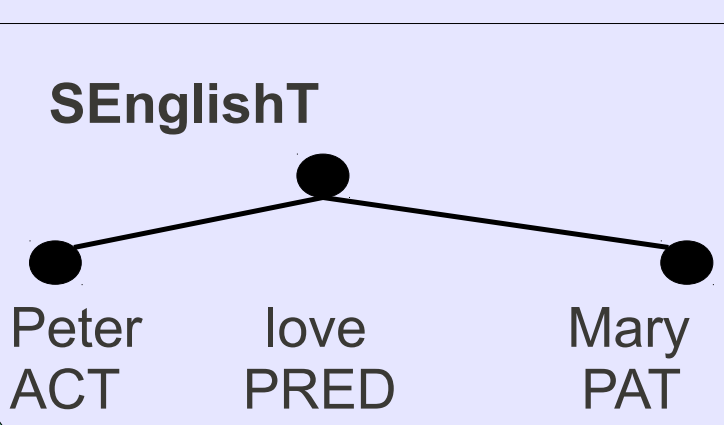

Czech layers

TCzechW Petr nemiluje Marii.

TCzechM

Petr milovat Marie NNMS1 VB-S-3P-NA NNFS4

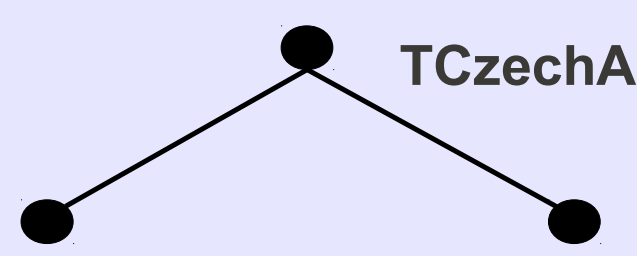

Petr milovat Marie

$\mathrm{Sb} \quad$ Pred Obj

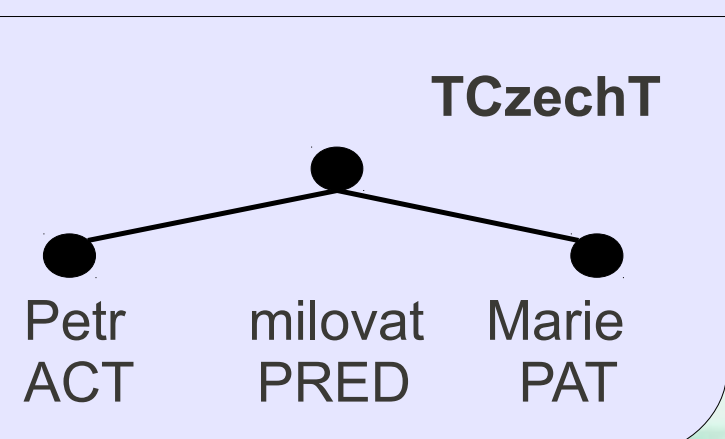

sentence 2

BUNDLE sentence $\mathrm{N}$

BUNDLE 


\section{TectoMT architecture data units}

\section{DOCUMENT}

sentence 1

English layers

\section{BUNDLE}

Czech layers

TCzechW

SEnglishW

Peter does not love Mary.

SEnglishM

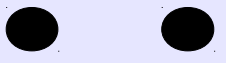

Peter do $n$ NNP VBZ $\mathrm{F}$

SEnglishA

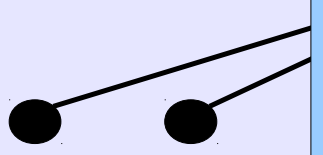

Peter do $n$

$\mathrm{Sb}$ AuxV N
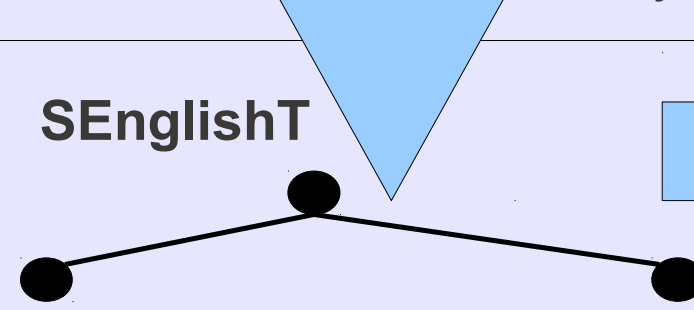

Peter

ACT love

PRED

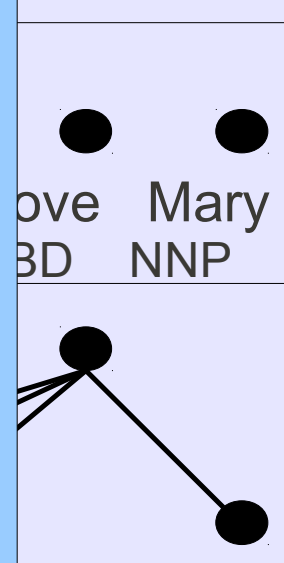

ove Mary

brad Obj sentence 2

BUNDLE sentence $\mathrm{N}$

BUNDLE 


\section{Internals - Design decisions}

- Perl (wrappers for binaries, Java,...)

- Linux (some applications platform-independent)

- OOP (ClassStd, Moose)

- Open source (GNU GPL for the versioned part)

- Neutral w.r.t. methodology (statistical, rule-based)

- Multilingual

- Open standards (Unicode, XML) 


\section{Internals - Components}

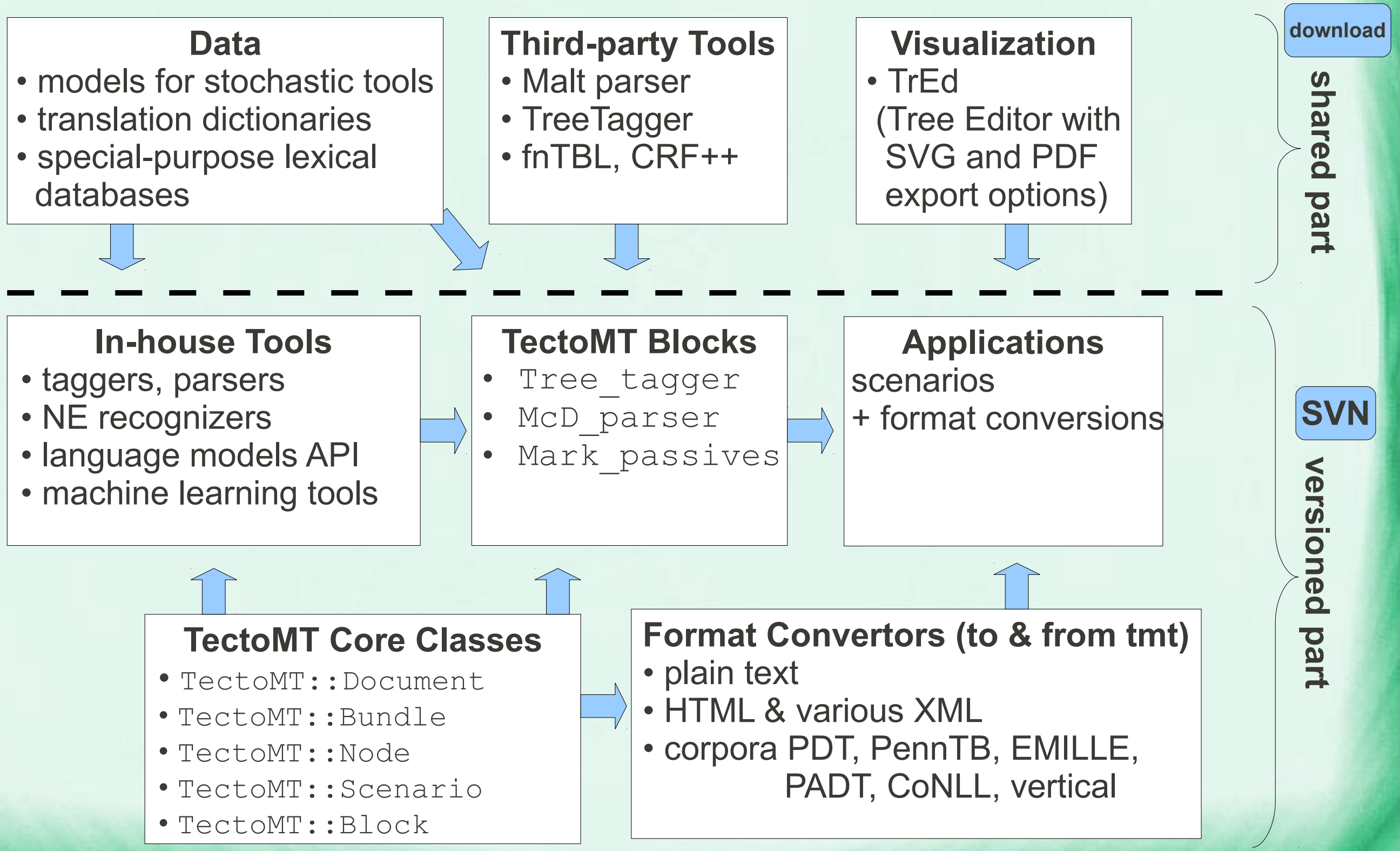




\section{Internals - Statistics}

- Developed since 2005, over ten developers

- Over 400 blocks (140 English, 120 Czech, 60 English-to-Czech, 30 other languages, 50 language independent)

- Taggers (5 English, 3 Czech, 1 German and Russian) Parsers (Dep. 2 English, 3 Czech, 2 German; Const. 2 English) Named Entity Recognizers (2 Czech,1 English)

- Speed example: Best version of English-to-Czech MT

1.2 seconds per sentence plus 90 seconds loading, with 20 computers in cluster: 2000 sentences in $4 \mathrm{~min}$ 


\section{Internals - Statistics}

- Developed since 2005, over ten developers

- Over 400 blocks (140 English, 120 Czech, 60 English-to-Czech, 30 other languages, 50 language independent)

- Taggers (5 English, 3 Czech, 1 German and Russian, Tamil) Parsers (Dep. 2 English, 3 Czech, 2 German; Const. 2 English) Named Entity Recognizers (2 Czech,1 English)

- Speed example: Best version of English-to-Czech MT

1.2 seconds per sentence plus 90 seconds loading, with 20 computers in cluster: 2000 sentences in $4 \mathrm{~min}$ 


\section{Future plans (TectoMT $\rightarrow$ Treex)}

- Reimplementation of core components

- CPAN release

- Adding new languages more easily

- Improved parallelization support

- Faster code, smaller files,... 


\section{Conclusion TectoMT main properties}

- emphasized efficient development, modular design and reusability

- stratificational approach to the language

- unified object-oriented interface for accessing data structures

- comfortable development 


\section{TrEd visualization}

\section{translation}

File Node $\underline{\text { Tree }} \underline{\text { View Macros Setup } \text { Help }}$
The firm began series production last November.
Sériovou výrobu firma rozjela loni v listopadu.
Firma začala výrobu řady poslední listopad.

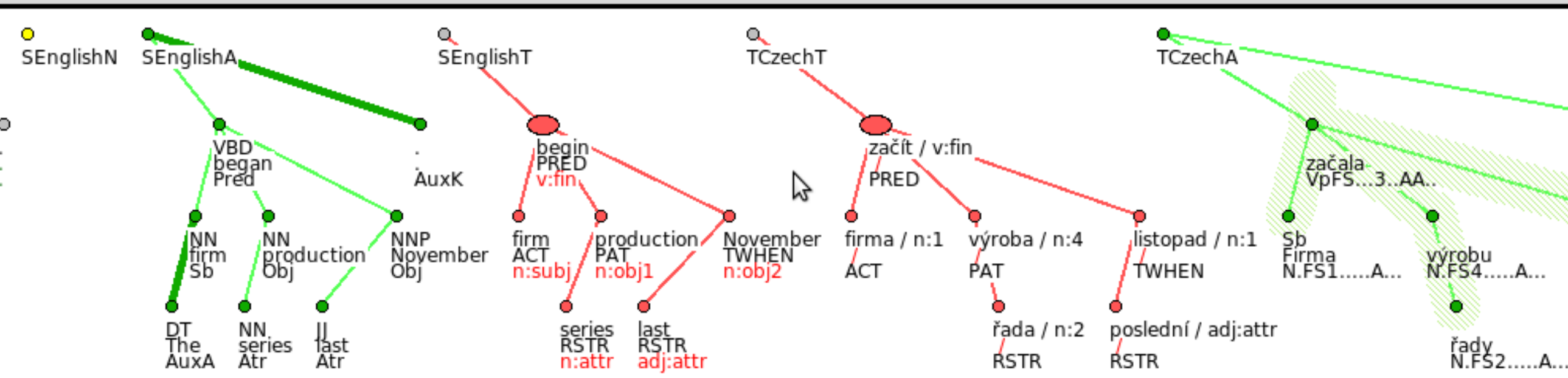




\section{TrEd visualization}

\section{word alignment on the morphological layer}

\section{SEnglishM}

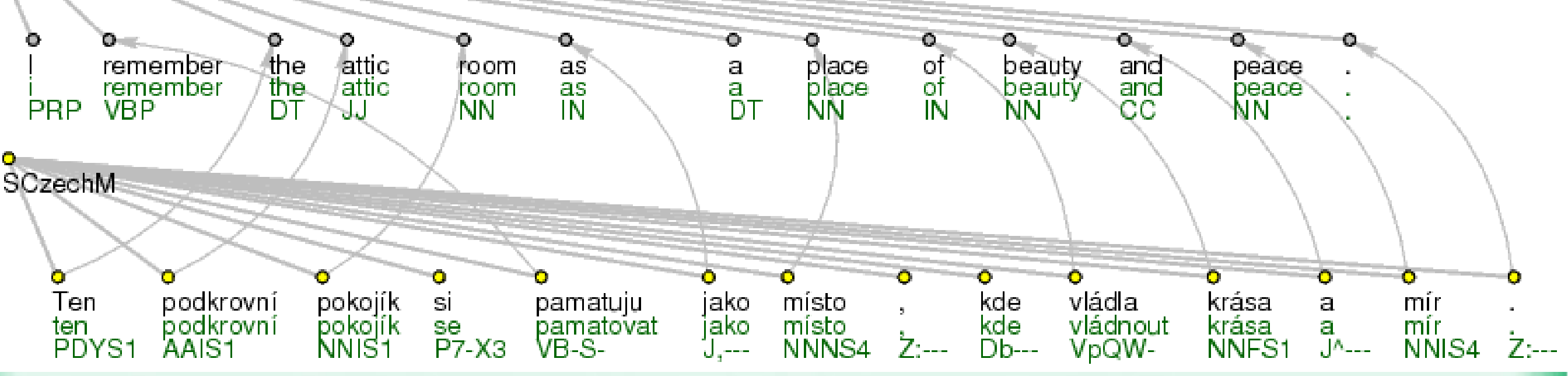




\section{TrEd visualization}

\section{word alignment on the tectogrammatical layer}

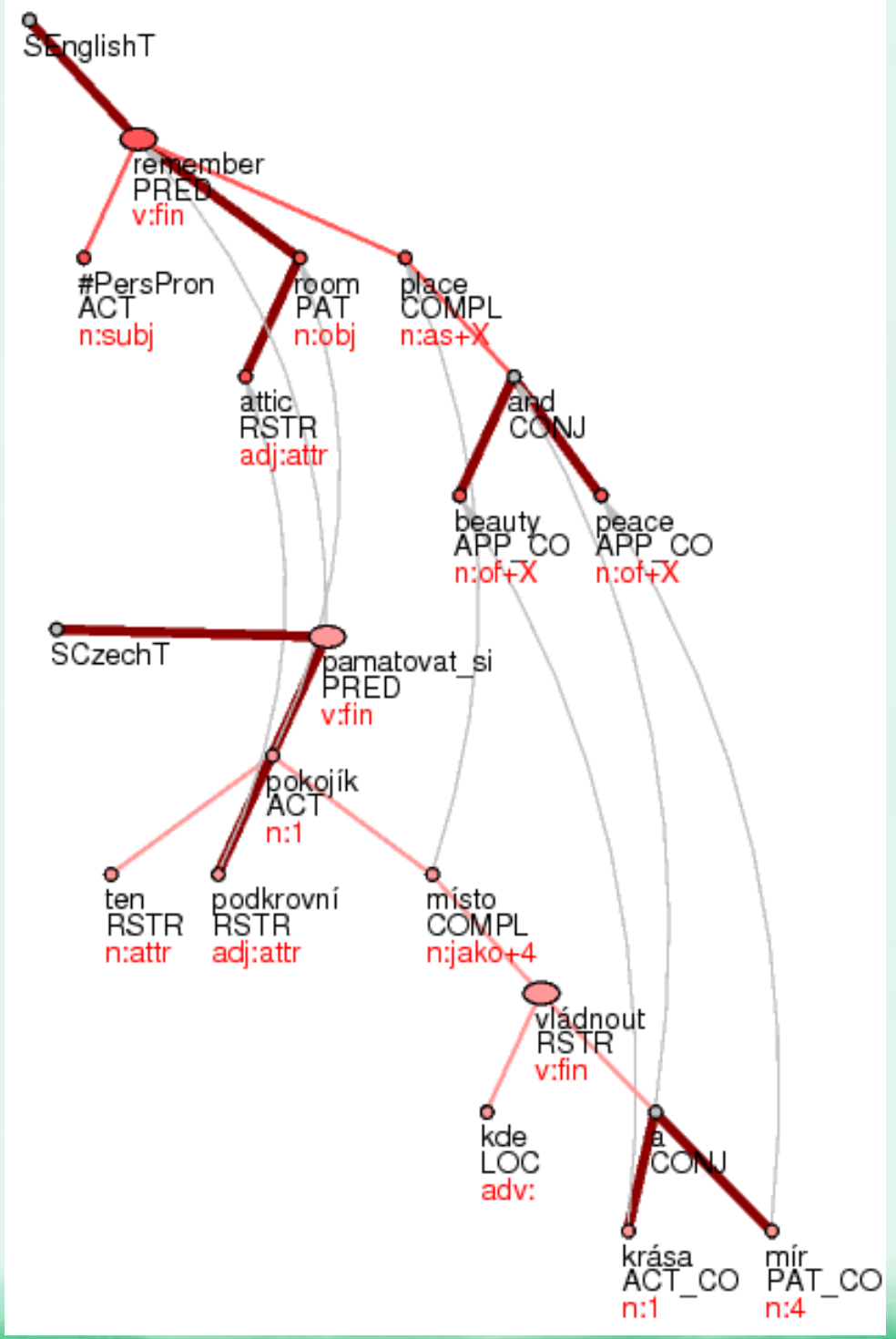




\section{Block example - SV}

use strict; use warnings;

use base qw(TectoMT::Block);

sub process_bundle \{

my (\$self, \$bundle ) = @_;

my $\$$ a_root $=$ \$bundle->get_tree('SEnglishA');

foreach my \$a_node ( \$a_root->get_descendants ()$)\{$

if ( \$a_node->get_attr ('m/tag') $=\sim / /^{\wedge} \mathrm{V} /$ ) $\{\quad$ \# verb found

foreach my \$child ( \$a_node->get_eff_children() ) \{

if ( \$child->get_attr('afun') eq 'Obj' ) \{\# object found \# Move the object and its subtree so it precedes the verb \$child->shift_before_node(\$a_node); \}

\}

\}

\} 


\section{Thank you}

\section{Cooperation is welcomed.}

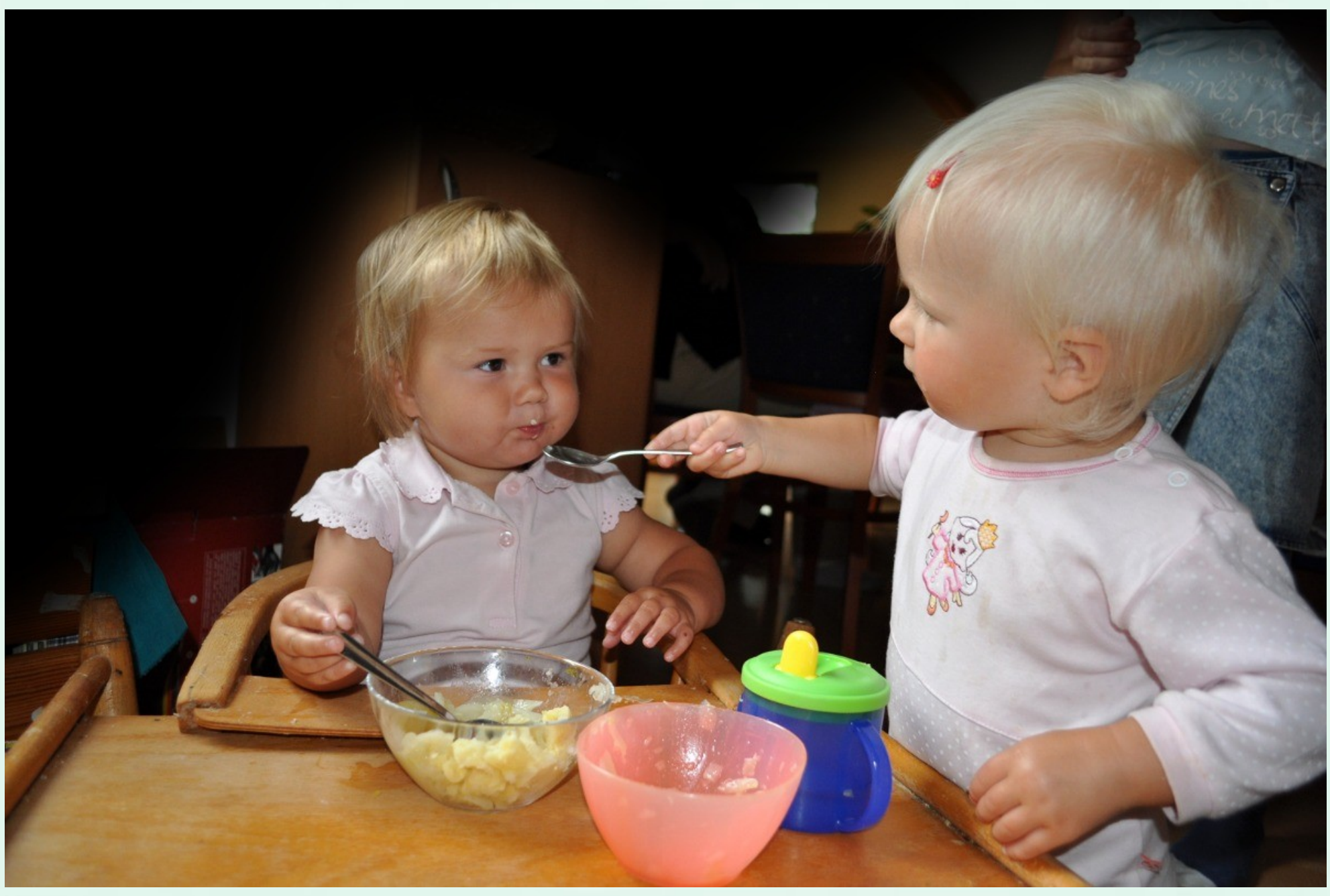

http://ufal.mff.cuni.cz/tectomt 